\title{
Selic e câmbio nominal nos próximos meses: a visão pelo modelo VAR com restrição
}

\author{
Luciano D'Agostini* \\ José Luís Oreiro**
}

Diante do cenário traçado pelo Banco Central do Brasil (BCB) sobre a atual conjuntura econômica, segundo a última ata do Comitê de Política Monetária (COPOM), faremos pelo método de Vetores-Auto Regressivos (VAR) as previsões das taxas de câmbio nominal e Selic para os próximos meses. Antes de apresentar as previsões da taxa de juros e câmbio, faremos três considerações: (i) mostrar a trajetória da taxa de juros e reservas internacionais do Brasil; (ii) mostrar a trajetória da taxa de juros dos Estados Unidos durante o período de adoção do sistema de metas de inflação no Brasil; (iii) mostrar o papel da taxa Selic no controle da inflação brasileira.

\section{Considerações sobre o câmbio nominal}

Comparando a taxa de juros de desconto dos títulos americanos (gráfico 1) com a taxa de câmbio nominal (gráfico 2) observa-se que existe uma relação negativa entre a taxa de juros americana e a taxa de câmbio nominal. Conforme o gráfico 1, quando a taxa de juros americana aumenta, o dólar se deprecia em relação ao Real. Um exemplo recente pode ser observado a partir de julho de 2004, quando ocorrem sucessivos aumentos das taxas de juros dos títulos do Tesouro norte-americano (2,25\% a.a. em julho de 2004 para 5,25\% a.a. em agosto de 2006).

A política monetária restritiva e de combate à inflação nos EUA, via aumento da taxa de juros, contribuiu para a depreciação do dólar, não somente em relação ao real, mas em relação as principais moedas internacionais. Quanto ao cenário da taxa de juros americana para os próximos meses, o Federal Open Market Committee (FOMC) sinalizou a proximidade do fim do ciclo da elevação das taxas de juros. Aqui o termo "proximidade" sugere aos analistas duas possíveis interpretações: (i) que os juros ainda podem aumentar e em um segundo momento finalizar a trajetória de alta; ou (ii) a alta dos juros realmente já chegou ao fim e iniciará, a partir da próxima reunião do FOMC, uma trajetória de queda.

\footnotetext{
* Doutorando em Desenvolvimento Econômico pela UFPR. Professor assistente da Faculdade Radial e do Instituto Brasileiro de Pesquisa e Pós-Graduação (IBPEX). Endereço eletrônico: lucianodagostini@yahoo.com.br

** Doutor em Economia pelo IE/UFRJ. Professor Adjunto do Departamento de Economia da UFPR e Pesquisador do CNPq. Endereço eletrônico: joreiro@ufpr.br Página pessoal: www.joseluisoreiro.ecn.br
} 
Gráfico 1. Taxa de juros de desconto dos títulos dos EUA (\% a.a. Jan/99 - ago/06)

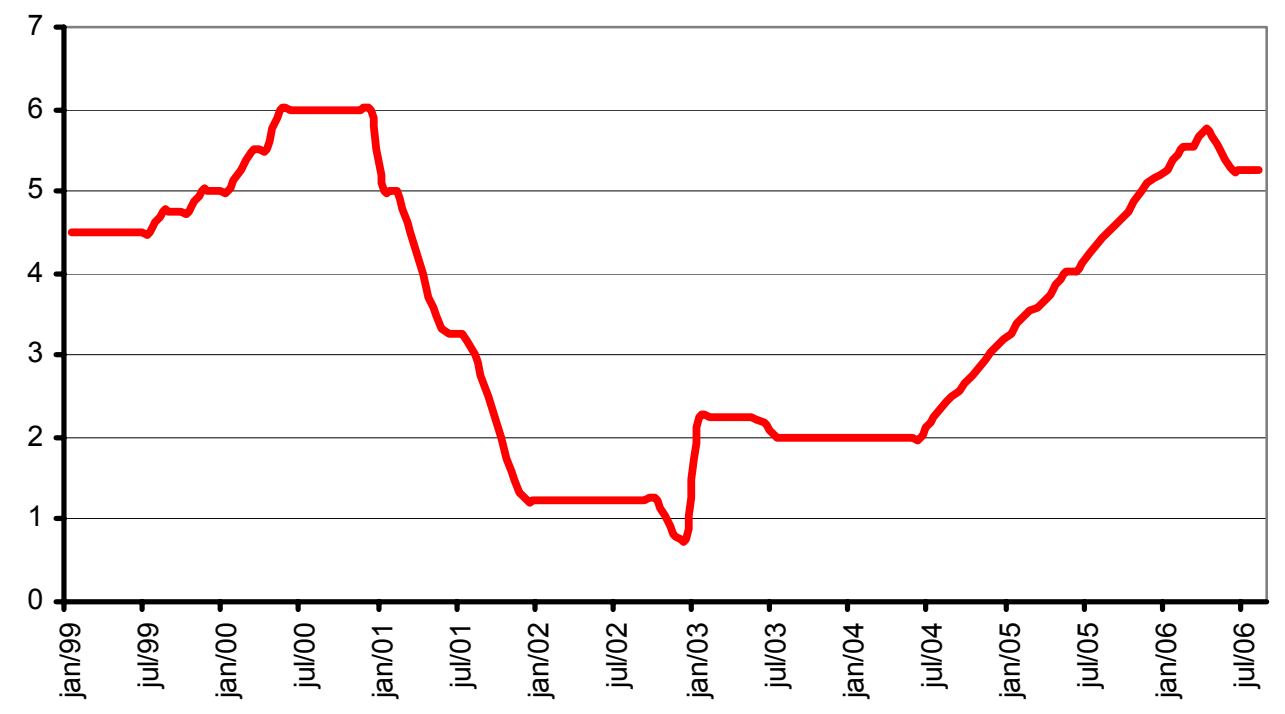

Fonte: Federal Reserve System

Pelo lado da economia brasileira, considerando os gráficos 3 e 4, destaca-se a trajetória das reservas internacionais e da Selic. Conforme o gráfico 3, a política de recomposição das reservas internacionais brasileiras, iniciada em 2003, também ajuda a explicar o comportamento de apreciação do real perante o dólar (Gráfico 2). Quando o câmbio estava em US $\$ 1,00=\mathrm{R} \$ 3,80$ em outubro de 2002, o Brasil obteve neste período um saldo positivo de US\$ 35,8 bilhões em reservas internacionais. Desde então, ocorreram sucessivos aumentos das reservas internacionais, combinados com apreciação do real, hoje situado em torno dos R \$2,15 por dólar (média de agosto/2006). Ao final de março de 2006, após a liquidação de US\$ 15,5 bilhões referentes à dívidas junto ao FMI, as reservas internacionais brasileiras alcançaram US $\$ 57,1$ bilhões (um aumento de 59,5\% em relação à outubro de 2002). De fevereiro a setembro de 2006, portanto, em apenas 7 meses, as reservas internacionais brasileiras aumentaram 29\% (de US $\$ 57,1$ bilhões para US\$ 73,7 bilhões). 
Gráfico 2. Taxa de câmbio nominal (Real/Dólar) - jan./99 a ago./06

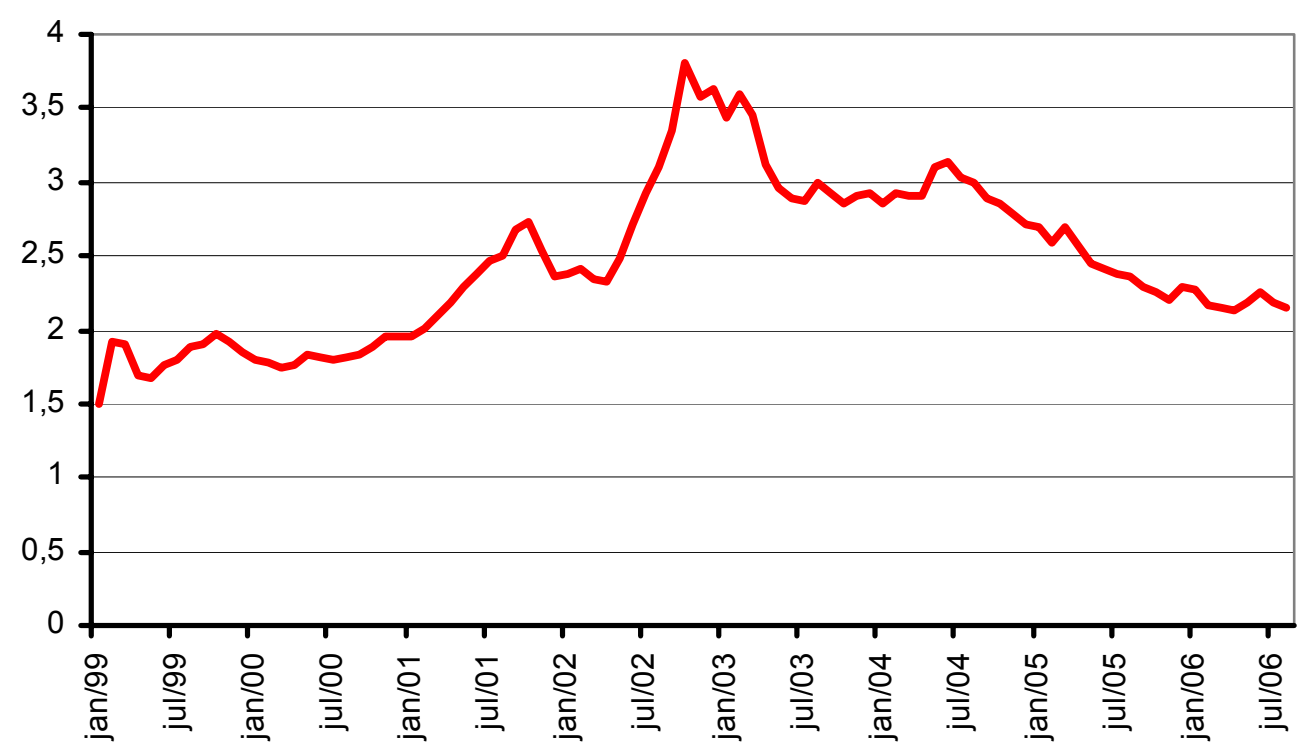

Fonte: Banco Central do Brasil

Gráfico 3. Reservas internacionais do Brasil (US\$ bi)

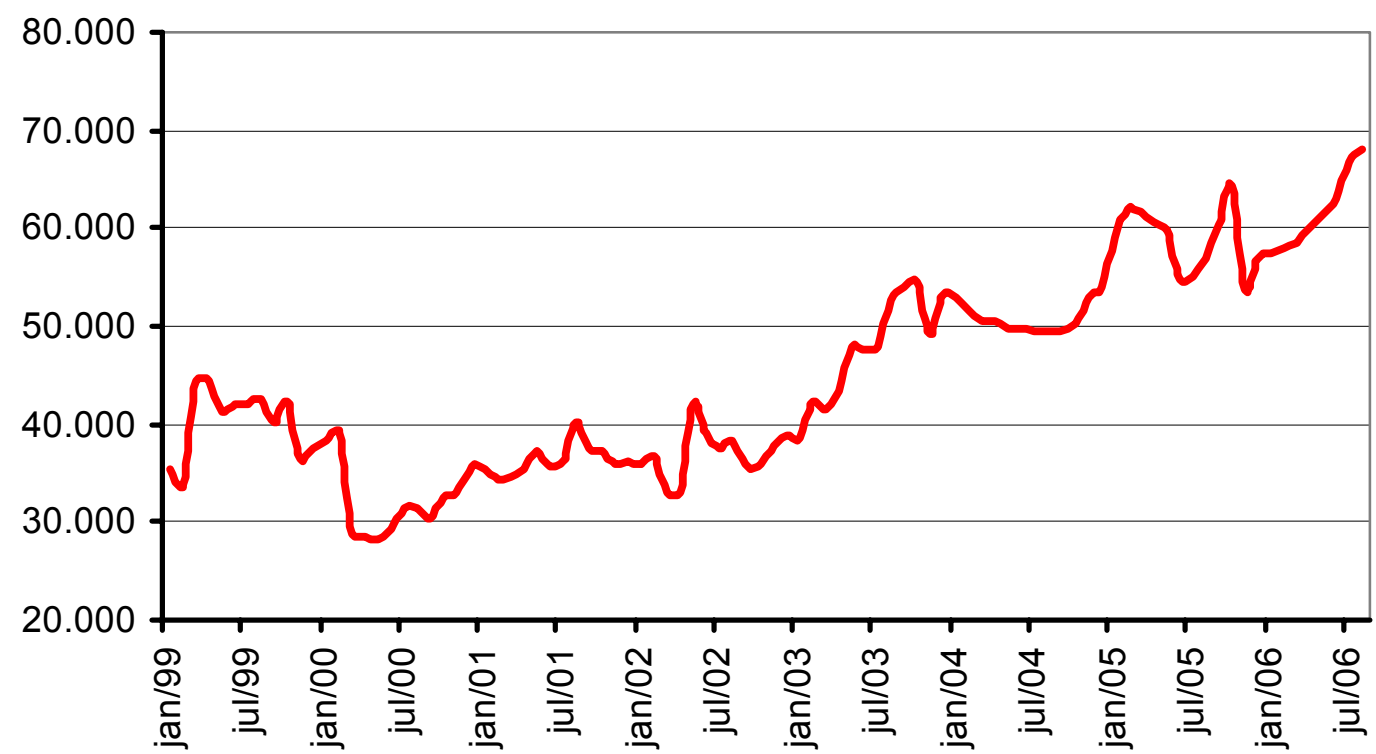

Fonte: BCB (Banco Central do Brasil)

No gráfico 3, pode-se observar que os sucessivos superávits comerciais, e a entrada de capitais estrangeiros ajudam a explicar o comportamento das reservas internacionais à disposição da economia brasileira. Outra variável que explica o comportamento do câmbio 
nominal é a taxa de juros Selic (gráfico 4). Durante o início da última trajetória de alta da Selic verificado em julho de 2004, quando estava em 15,75\% a.a, até o fim do processo em agosto de 2005 , quando estava em 19,75\% a.a, observou-se uma queda do volume dos meios de pagamentos dado por M1. No mesmo período, aumentou o volume de moeda pelo conceito de M2 (este conceito incorpora os títulos da dívida que rendem juros Selic).

Com relação ao balanço de pagamentos, a Selic agiu de forma positiva na conta capital financeira, propiciando entrada de capitais, pois os agentes econômicos, ao comparar as taxas reais de ganho e o risco macroeconômico de outros países, perceberam vantagens para investir no mercado de capitais brasileiro. Desde então, o risco-país caiu (o Emerging Market Bond Index - EMBI+ fechou em 223 pontos em agosto/2006). Tais considerações sobre a entrada de capitais e o aumento da Selic contribuíram também para apreciar o real frente ao dólar.

\section{Gráfico 4. Taxa de juros Selic no período jan./99 a ago./06 (em \%)}

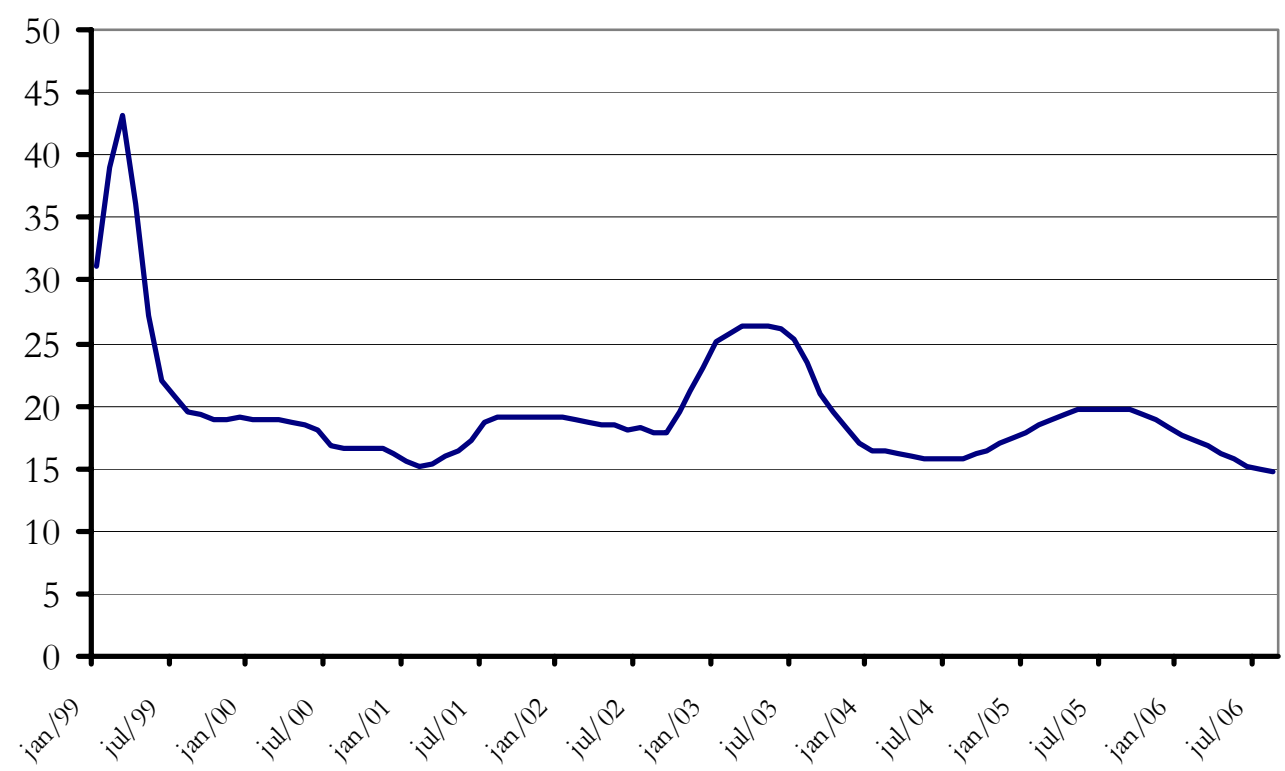

Fonte: Banco Central do Brasil

A taxa de câmbio nominal, série 3697 do BCB (Gráfico 2), caiu de R 3,12 em junho de 2004 para os atuais $\mathrm{R} \$ 2,15$ em agosto de 2006, representando uma queda de 31,1\%. Diante do objetivo do governo brasileiro de manter a inflação sob controle aliado ao aumento das reservas cambiais, e do cenário da economia norte-americana, o que acontecerá com a taxa de câmbio nominal real/dólar nos próximos meses? 
Em outras palavras, será que a economia norte-americana, por ser maior que a do Brasil, exercerá um papel determinante para continuar o processo de depreciação do dólar perante o real, a partir de sua conduta de política monetária? Será que a queda da taxa de juros Selic terá impactos para que a moeda nacional se deprecie? Será que o aumento do volume de reservas internacionais brasileiras continuará a exercer um papel importante para que o real continue se apreciando?

\section{Resultados da previsão do câmbio nominal e Selic}

A estimação da Selic será determinada com base na avaliação dos valores correntes e futuros de variáveis da economia brasileira, tais como produto, moeda, câmbio e preços. $\mathrm{Na}$ estimação da taxa de câmbio nominal incluiremos no VAR a taxa de juros norte-americana e as reservas internacionais do Brasil. Portanto, para a previsão da Selic utilizam-se 5 variáveis e para a previsão da taxa de câmbio nominal, 7 variáveis.

Com dados mensais, de janeiro de 1999 a agosto de 2006, inclui-se no modelo VAR, as seguintes variáveis: para preços, adotou-se o IPCA (código 433 do IBGE) por ser a taxa de inflação utilizada para estabelecer a meta de inflação. Para a taxa básica nominal de juros, utilizou-se a Selic (código 4189 do BCB), principal instrumento de controle da inflação. Para o produto adota-se o PIB mensal em valores correntes, medida em milhões de reais (código 4380 do BCB). A série de moeda é M2, código 1837, a série de câmbio nominal é a 3697 e a série de reservas internacionais brasileiras, pelo conceito de liquidez total, é a 3546, ambas extraídas do BCB. Por fim, a taxa de juros norte-americana, que é a taxa de desconto do Banco Central dos Estados Unidos, extraída do FED.

Assumindo endogeneidade nas variáveis do sistema, a taxa de câmbio nominal e a Selic podem ser estimadas consistentemente pelo VAR. Os procedimentos econométricos, encontrados em D’Agostini-Sampaio ${ }^{25}$ (2006), estão resumidos nas seguintes etapas: (i) Logaritimizar as séries; (ii) Efetuar teste de raiz unitária pelo ADF; (iii) Efetuar os testes de Cointegração Johansen-Juselius (JJ); (iv) Escolher a defasagem ótima do VAR pelo critério de Akaike (AIC) e Schwartz (SBC); (v) Conforme o teste JJ adota-se o modelo VAR simples ou com correção de erro (VECM); (vi) determinar os coeficientes das equações por MQO; (vii) observar a equação de equilíbrio de longo prazo entre as variáveis, dada pelo VECM; (viii)

${ }^{25}$ D’Agostini, L. L M.; Sampaio, A.V. (2006). Monismo Versus Não Monismo no Brasil. Encontro de Economia da Região Sul (ANPEC-SUL 2006). 
substituir os valores presentes e passados na equação da taxa de câmbio e na equação da taxa de juros.

\section{Resultados}

Os resultados da previsão da taxa de juros estimada a partir de um VAR(4) com correção de erros, estão dispostos na tabela 1 e a previsão da taxa de câmbio nominal, estimada a partir de um $\operatorname{VAR}(3)$ com correção de erros está na tabela 2.

Tabela 1. Comparação da taxa Selic efetiva com a estimada em \% a.a.

\begin{tabular}{cccc}
\hline Mês & Selic Efetiva \% a.a. & $\begin{array}{c}\text { Previsão Selic } \\
\text { pelo VAR }\end{array}$ & Elasticidade Juros \\
\hline Set $/ 06$ & $14,25 \%$ & - & -0.0256731229567 \\
Out/06 & $?$ & $13,88 \%$ & -0.0147917323057 \\
Nov $/ 06$ & $?$ & $13,67 \%$ & -0.0158562842248 \\
Dez $/ 06$ & $?$ & $13,45 \%$ & - \\
\hline
\end{tabular}

Fonte: Banco Central do Brasil e Dados da Pesquisa.

Tabela 2. Previsão da taxa de câmbio nominal (R\$/US\$) pelo VAR

\begin{tabular}{cccc}
\hline Mês & Câmbio Efetivo & $\begin{array}{c}\text { Previsão Câmbio } \\
\text { Nominal** }\end{array}$ & $\begin{array}{c}\text { Elasticidade } \\
\text { Câmbio }\end{array}$ \\
\hline Ago/06 & 2,1551 & - & 0.0201035822774 \\
Set/06 & $?$ & 2,1984 & 0.0102673987143 \\
Out/06 & $?$ & 2,2209 & -0.0268598484183 \\
Nov/06 & $?$ & 2,1612 & - \\
\hline
\end{tabular}

Fonte: Banco Central do Brasil e Dados da Pesquisa.

Os resultados das tabelas 1 e 2 mostram uma provável queda na taxa de juros Selic e uma pequena depreciação do real frente ao dólar para os próximos meses. Para o mês de outubro o modelo indica a previsão da Selic próxima a 13,88\% a.a. Do mesmo modo, pela análise da elasticidade juros, as previsões para novembro e dezembro são de 13,67\% e 13,45\% a.a., respectivamente. Isto sugere que a Selic até o fim do ano deve alcançar 13,5\% a.a.

O Copom irá realizar ainda duas reuniões em 2006. A penúltima nos dias 17 e 18 de outubro e a última nos dias 28 e 29 de novembro. Como o modelo VAR aqui apresentado prevê uma Selic de 13,88\% a.a. para outubro e dada a prática do BCB em reduzir a taxa de juros em intervalos de $0.75,0.5$ ou 0.25 p.p. a cada reunião, segue-se que nossa previsão é que o Copom reduza a meta para a Selic em 0.5 p.p na reunião de outubro, levando assim a taxa nominal de juros para $13.75 \%$ a.a.

O cenário para a reunião de novembro não é tão claro. Isso porque a previsão da Selic para o mês de novembro é de $13.67 \%$ a.a, um valor muito próximo de nossa expectativa 62 
para a mesma variável em outubro. Dessa forma, o modelo estaria indicando que o BCB interromperia o processo de redução da taxa de juros na reunião de novembro. Contudo, a previsão do modelo aqui apresentado para o mês de dezembro é de $13.45 \%$ a.a de juro nominal. Sendo assim, acreditamos que o Copom também deverá reduzir a meta de taxa de juros na última reunião do ano. Nesta, a redução seria mais comedida: o Copom deve reduzir a Selic em 0.25 p.p levando o juro nominal para o patamar de $13.5 \%$ a.a em sua última reunião do ano de 2006.

No mais, o resultado da taxa de câmbio nominal, com a previsão de queda da Selic, indica que o real terá um movimento de pequena depreciação perante o dólar, nos meses de setembro e outubro, retomando o processo de valorização em novembro. Para outubro a previsão do câmbio é US $\$ 1,00=\mathrm{R} \$ 2,22$; e para novembro $\mathrm{R} \$ 2,16$.

\section{Anexo}

A equação (1) de previsão da taxa de juros simulada é:

$\mathrm{D}(\mathrm{LJB})=-0.01564509878 *(\mathrm{LJB}(-1)-14.97080933 * \mathrm{LM1}(-1)+1.837236385 * \mathrm{LCAMBIO}(-1)-$ 0.6938080055*LIPCA $(-1)+1.897675695 * \operatorname{LPIB}(-1)+0.1556274805 * @$ (TREND(99:02)) + 238.4749662 ) + $0.5575817526 * \mathrm{D}(\mathrm{LJB}(-1))-0.1641472192 * \mathrm{D}(\mathrm{LJB}(-2))+0.01504719053 * \mathrm{D}(\mathrm{LJB}(-3))-0.01605733261 * \mathrm{D}(\mathrm{LJB}(-$ 4)) - $0.1878543928 * \mathrm{D}(\operatorname{LM} 1(-1)) \quad-\quad 0.1759413806 * \mathrm{D}(\mathrm{LM} 1(-2)) \quad-\quad 0.09573765166 * \mathrm{D}(\operatorname{LM} 1(-3)) \quad$ $0.04210090753 * \mathrm{D}(\mathrm{LM} 1(-4))+0.07557198674 * \mathrm{D}(\mathrm{LCAMBIO}(-1))+0.00566594496 * \mathrm{D}(\mathrm{LCAMBIO}(-2))+$ $0.1166867439 * \mathrm{D}(\mathrm{LCAMBIO}(-3))+0.1035521847 * \mathrm{D}(\mathrm{LCAMBIO}(-4)) \quad-\quad 0.007756335253 * \mathrm{D}(\mathrm{LIPCA}(-1)) \quad$ $0.002049777833 * \mathrm{D}(\mathrm{LIPCA}(-2)) \quad-0.002219049483 * \mathrm{D}(\operatorname{LIPCA}(-3))+0.002165209164 * \mathrm{D}(\operatorname{LIPCA}(-4)) \quad+$ $0.09452555478 * \mathrm{D}(\operatorname{LPIB}(-1))+0.08667414166 * \mathrm{D}(\operatorname{LPIB}(-2))+0.07227986098 * \mathrm{D}(\mathrm{LPIB}(-3))+$ 0.09539434572*D(LPIB(-4)) - 0.0001569040327 + 7.388529038e-06*(@TREND(99:02))

A equação (2) de previsão da taxa de câmbio nominal simulada é: $\mathrm{D}(\mathrm{LCAMBIO})=-0.001026229298 *(\mathrm{LJE}(-1)-10.03024664 * \mathrm{LCAMBIO}(-1)-4.406512311 * \mathrm{LJB}(-1)+$ $52.88694513 * \operatorname{LM1}(-1)+6.72043759 * \operatorname{LIPCA}(-1)+3.414421099 * \mathrm{LRB}(-1)+39.67194994 * \operatorname{LPIB}(-1)-$ 0.9947168425*@TREND(99:02)) - 1392.511459 ) + 0.04678831486*D(LJE(-1)) - 0.01371136528*D(LJE(-2)) $0.06413454386 * \mathrm{D}(\mathrm{LJE}(-3))+0.335644153 * \mathrm{D}(\mathrm{LCAMBIO}(-1))+0.08153094601 * \mathrm{D}($ LCAMBIO $(-2))+$ $0.1338351149 * \mathrm{D}(\mathrm{LCAMBIO}(-3)) \quad-\quad 0.1310767453 * \mathrm{D}(\mathrm{LJB}(-1)) \quad+\quad 0.04065122163 * \mathrm{D}(\mathrm{LJB}(-2)) \quad$ $0.03954442689 * \mathrm{D}(\mathrm{LJB}(-3))-0.0255360614 * \mathrm{D}(\mathrm{LM} 1(-1))-0.02571830898 * \mathrm{D}(\operatorname{LM} 1(-2))-0.1160844131 * \mathrm{D}(\mathrm{LM} 1(-$ 3) $)+0.0001617455639 * \mathrm{D}(\operatorname{LIPCA}(-1))+0.001466521538 * \mathrm{D}(\operatorname{LIPCA}(-2))+0.008375076141 * \mathrm{D}(\mathrm{LIPCA}(-3))+$ $0.04250639461 * \mathrm{D}(\mathrm{LRB}(-1)) \quad-\quad 0.0007577718464 * \mathrm{D}(\mathrm{LRB}(-2)) \quad+\quad 0.03819341975 * \mathrm{D}(\mathrm{LRB}(-3)) \quad-$ $0.27091149 * \mathrm{D}(\operatorname{LPIB}(-1))+0.191429789 * \mathrm{D}(\operatorname{LPIB}(-2))-0.0330522917 * \mathrm{D}(\operatorname{LPIB}(-3))+0.01071852495-$ 0.000160692885*(@REND(99:02)) 
Onde $\mathrm{D}($.$) é a primeira diferença; (-1), (-2)..., (-4) são as defasagens das variáveis$ utilizadas no modelo e; @TREND (.) é o componente de tendência.

Os modelos VAR são do tipo Log-Log. Efetuando as substituições dos valores das variáveis defasadas no sistema, para diversos períodos de tempo, temos o valor das elasticidades dos juros e do câmbio. Estas elasticidades determinam, para os períodos $t+1$, $\mathrm{t}+2, \ldots, \mathrm{t}+\mathrm{n}$, os valores das previsões das taxas de juros e câmbio subsequentes.

Por questões probabilísticas do modelo, quanto maior é o tempo de previsão menor é a possibilidade de acerto. Portanto, o modelo VAR serve apenas para previsões de curto prazo. Por isso, nas Tabelas 1 e 2, mostra-se os valores esperados da Selic e câmbio nominal para 4 e 2 períodos à frente, respectivamente. 